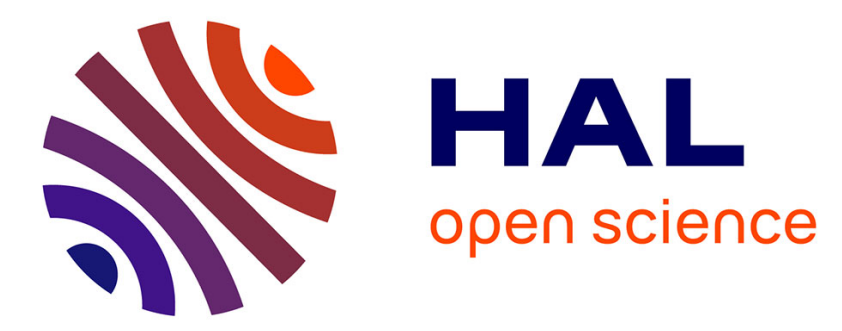

\title{
Addressing Constraints Creatively: How New Design Software Helps Solve the Dilemma of Originality and Feasibility
}

\author{
Pierre-Antoine Arrighi, Pascal Le Masson, Benoit Weil
}

\section{To cite this version:}

Pierre-Antoine Arrighi, Pascal Le Masson, Benoit Weil. Addressing Constraints Creatively: How New Design Software Helps Solve the Dilemma of Originality and Feasibility. Creativity and Innovation Management, 2014, pp.10.1111/caim.12082. 10.1111/caim.12082 . hal-01083248

HAL Id: hal-01083248

https://hal-mines-paristech.archives-ouvertes.fr/hal-01083248

Submitted on 17 Nov 2014

HAL is a multi-disciplinary open access archive for the deposit and dissemination of scientific research documents, whether they are published or not. The documents may come from teaching and research institutions in France or abroad, or from public or private research centers.
L'archive ouverte pluridisciplinaire HAL, est destinée au dépôt et à la diffusion de documents scientifiques de niveau recherche, publiés ou non, émanant des établissements d'enseignement et de recherche français ou étrangers, des laboratoires publics ou privés. 


\title{
Addressing constraints creatively: how new design software helps solve the dilemma of originality and feasibility
}

\author{
Pierre-Antoine Arrighi, Pascal Le Masson and Benoit Weil
}

Are designers doomed to sacrifice creativity when integrating new product development processes? Although many studies highlight the need to produce original and innovative designs, maintainingcreativity in the design process continues to be difficult due to industrial constraints. Thus, creativity is restricted to phases in the "Fuzzy Front End" to avoid those constraints that might effectively kill it(Amabile, 1998, Reid and De Brentani, 2004). However, constraints are also acknowledged as a resource for creativity, ashas previously been shown with artists and engineers (Burkhardt and Lubart, 2010, Sternberg and Lubart, 1999, Le Masson et al., 2011, Goldenberg and Mazursky, 2000).Thus, we posethefollowing research question: In which cases can a constraint be a resource for creativity? To answer this question, we investigate different types of computer-aided design (CAD) software. Relying on an experimental method, we compare the performance of those types of software at the so-called ideation gap where design sketches are transformed into digital models. We show the following: 1) some CAD software enables designers to work under additional constraints and be more creative and toavoid the tradeoff between robustness and creativity,and 2) understanding this performance means appreciating that such software enables designers to play with the embedded constraints to revealassociated fixations and to design models that follow the constraint but overcome the fixation. Constraints and creativity are linked by two competing processes: constraints decrease the degree of freedom and, as a result, creative possibilities, but embedding constraints increases the awareness of fixationsandtherefore the capacity to design original models. Today, new CAD tools more effectively support the second process, which leads to "acquired originality" in design.

'What is not constrained is not creative.' Philip Johnson-Laird

\section{Introduction}

It is wellknown that creativity involves not only originality but also feasibility, i.e. not only "good ideas" but also feasible ones. However, creative phases frequentlyresult in conflict with the feasibility constraints of the industrial environment. The challenge for firms is to create a context in whichcreative people and activitiescangenerate (creative) outcomes while guaranteeing that their work is compatible with and can be assimilated into the industrial design process. Can creative phases be integrated into an industrial design process without damaging or restrainingoriginality? The literature describes different approaches to and advice for combining creativity and industrial constraints in design with respect to issues such as fabrication, costs and the environment. However, there is a continuing debate regarding the best time to integrate and exploit innovative potential during the new product development (NPD) process. 
For some researchers, creativity must be fostered at the beginning of the design process. This approach, first popularized by scientists working primarily in automobile design (Fujimoto, 1997, Thomke and Fujimoto, 2000), is now proposed by publications that take the "Fuzzy Front End" approach to NPD (Khurana and Rosenthal, 1998, Reid and De Brentani, 2004). Following the hypothesis that conforming a design to industrial constraints impedes creativity, the Fuzzy Front End approach advocates for increasingthe creative potential before addressingconstraints. In this scheme, the best guarantee for high levels ofcreativity appears to be maximum freedom at the beginning of the design process(Karniel and Reich, 2011).The NPD process then consumes this initial creativity potential. Under this approach, there is a tension between creativity (which is considered as a number of degrees of freedom) and constraints (which reduce thedegrees of freedom and should be introduced later in the process). Thus, there is a trend in the creativity and innovation management literature to posit an opposition between creativity and constraint, i.e., between originality and robustness.

A different community introduces the design process as a succession of iterative steps that all require the creative capacity to challenge previous choices and to continuously "reframe the problem" with creativity. Donald Schön (Schön, 1983, Schön, 1990)was one of the first to consider design to be more than a problem-solving activity in that managing constraints during the design process should be coupled with creativity and originality. Constraints can trigger innovation and lead to original and creative design. This concept is understood byartists (writers, poets, painters, etc.) who use constraints to increase their creativity, such asthe French literary movement known as "Oulipo" ("Ouvroir de littérature potentielle", which is roughly translated asthe "workshop of potential literature"), in which creativity is stimulated by writing constraints (e.g.,using particular words, avoidingcertain letters,etc.). This concept might also apply to engineering design. It has been shown that recent design theories tend to support the dual improvement in robustness and generativeness, whichleads to interweavingthese terms in design processes (Le Masson et al., 2011).

Thus, there is a research gap in the creativity and innovation literature:are these two types ofperformances - generativeness and robustness - necessarily evolving in opposite directions during NPD, or is it possible for them to grow simultaneously during NPD? If the latter is true, can we identify some of the conditions for creatively addressing constraints to solve the dilemma between generativeness and robustness?

To bridge this complex research gap, we focus specifically on computer-aided design (CAD) softwaretools. CADtools are thedominant means ofproduction in the design field, and examining their use can yield vivid evidence regardingthe design process. Today, CAD tools confront the tension between originality and feasibility when engaging withthe so-called "design gap"(Wendrich et al., 2009), i.e., the loss of originality that accompanies coding design sketches into digital models in CAD suites. We compare the capacities of two different generations of CAD tools in term of generativeness and robustness (i.e., increase or loss of originality and increase or loss of feasibility). Both tools, which belong to the same software design suite, are used to generate numerical models. The first CAD tool tends to apply as few constraints as possible to the shape design process to correspond as faithfully as possible to the initial sketch. Shapes are generated through process operations (e.g., blueprints, extrusions, etc.). A second CAD tool immediately embeds certain constraints (on optical quality) on the shape and relies on a new shape deformation process, which is closer to the paradigm of clay deformation. Thus, the two tools 
differ in how they address constraints. After the shapes are generated, we assess the respective effects that using each of these two tools has on the robustness and generativeness of concepts during this design step.

We first present the theoretical background underlying our research hypotheses. We then detail our experimental plan with a presentation of our experimental logic, the variables and the measures that we used in the experiment. This introduction is followed by sections that present the analysis of the tools' performances, discuss our results and offer our conclusions.

\section{Motivations, Theoretical Background and Hypothesis}

Many studies have shown that creativity and originalityare important to triggering, fostering and sustaining innovation (Olson et al., 1998, Verganti, 2008).However, creativity and innovation capacities are difficult to integrate into industrial environments. The challenge for firms is to support originality and "outside-the-box" thinking while guaranteeing that such creativework is compatible with and can be assimilated into the industrial design process. Can this apparently direct trade-off between creativity and constraint be resolved? That is, can creative activities be integrated into an industrial design process without damaging or restraining the objective of obtaining original output?

\section{A divided literature regarding management of creativity and industrial constraints}

Certain researchers have found that it is necessary to foster creativity at the beginning of the design process. This approach was first popularized by scientists working primarily in the field of automobile design (Midler, 1995) (Fujimoto, 2007).

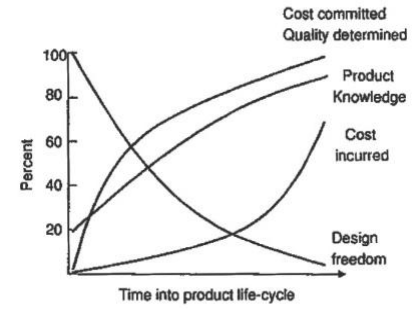

Figure 1. Product knowledge and design freedom versus time (Karniel \& Reich, 2011)

Karniel and Reich (Karniel and Reich, 2011)advocate forthis approach, based on the work of Ullman (Ullman, 1997). When creating a new design, there is a progressive trade-off between the degree of freedom in a project (which is also constrained by the remaining possibilities of action) and designers' knowledge regardingtheproject. The degree of freedom is directly linked to various means of exploration (also called possibilities) that have been preserved to enable innovation and that can be used to resolve the issues encountered. This freedomis consumed like capital as the design process progresses. This type of analytical approach ishighlighted in publications that emphasizethe Fuzzy Front End approach to NPD(Khurana and Rosenthal, 1998). A Fuzzy Front End approach also requires a phase of maximum creativity at the beginning of the design process that should be free from constraints to enable consideration of the widest range of solutions possible, according to Reid and de Brentani (Reid and De Brentani, 2004). These recommendations are intended to promotethe ultimate convergence toward a 
successful design because all the unpredicted future issues can be anticipated by preservingthe maximum degree of initial freedom. Creativity is consumed during the early phases; as options become progressively out of reach, freedom decreases. Thus, there is a tension between the freedom of creativity and the constraints of practical development.

Other scholars consider the design process as a succession of iterative steps, in which each step requires the capacity to challenge previous choices and to continuously and creatively "reframe the problem". In Van de Ven's "Innovation Journey" (Van de Ven et al., 1999), intensive and radical innovation is achieved by means ofa succession of iterative (and sometimes creative) steps. In other words, creativity is not always accomplished at the beginning of the innovation journey.Donald Schön (Schön, 1983, Schön, 1990), who was one of the first to consider design as more than a problem-solving activity, considers the design issue to consist of 'framing' and reframing a problem, and creative framing is constructed from the materials of problem situations. Schön also posits that each move is a local experiment that contributes to a global experiment in reframing the problem. Under this framework, managing constraints during the process is coupled with creativity and originality. This approach is widespread in many research communities and recommendsincluding creative phases throughout the design process (Couger, 1990, Dorst and Cross, 2001)). Buijs (Buijs, 2003)uses the expression "circular chaos," rather than "linear logic,"to refer to the design process.Some authors stress that a shift in a creative concept can occur even late in the design process (Seidel, 2007). Certain methods, such as TRIZ (sometimes defined as the "theory of inventive problem solving"), are supposed to creatively address constraints (Moehrle, 2010), whereas some tools, such as CAD software, enable the exploration of alternatives and preserve room for innovation throughoutthe design process(Thomke and Fujimoto, 2000).

Thus, there can be a form of complementarity between constraints and creativity.

\section{Deciphering from a design theories perspective}

To understand why there are such contradictory propositions, it is important to clarify the analytical framework. On the one hand, the tension between creativity and constraint arises out of an analysis of the development process as a problem-solving issue. In a problem-solving framework, constraintsindicate less freedom and creativity and are directly linked to the degrees of freedom, which means that there is an inherent conflict between creativity and constraints. On the other hand, it has long been shown that problem solving takes a view of design processes that is too limited (Schön, 1990, Rittel, 1972, Hatchuel, 2002). For example, from the broader perspective of design theory, a design process can consist of "reframing the problem" or, more generally, of generating original products and services that embody newly invented performance criteria. This concept describes the logic of originality in creative design: the outcome is not a "solution" to a problem but is original precisely because it cannot be deduced from a given set of rules (Boden, 1990). Contemporary design theories compete to model a growing number of generative processes, i.e., outside-the-box processes that extend beyond problem solving. In general terms (see the synthesis in (Hatchuel et al., 2011)), all these processes can be characterized by their capacity to generate design propositions from a given knowledge set, which is a process that can be characterizedby two criteria:

i) generativeness, i.e., the ability to produce design proposals that differ from existing solutions and design standards; and

ii) robustness, i.e.,the ability to produce designs that meet constraints (such as functional requirements, feasibility, production constraints, legal constraints,etc.). 
Together, these theories form a consistent body of knowledge that aimsto enhance the generativeness of a design without losing its robustness. This approach can be called a "simultaneous solution" of the two criteria. Well-known design theories-including General Design Theory (Yoshikawa, 1981), Axiomatic Design (Suh, 1990) and Concept-Knowledge (Hatchuel and Weil, 2009) - recommend that practitioners take these two dimensions into accountand manage them simultaneously throughout the entire design process to develop productsthat are both original and feasible (i.e., that function under theapplicable constraints).Therefore, there can be a positive relationship between robustness and originality, at least in theory.

Thus, within the framework of design theory, there are actually two competing phenomena:

- On the one hand, if they are accepted and met in the final output, constraints will continue tolower degrees of freedom, such as in problem solving.

- On the other hand, constraints can increase originality by leading to design outcomes that differ from existing solutions.

These two phenomena are present in design processes, and their balance might explain why some design processes take the form of a tradeoff between generativeness and robustness, whereas other processes appear to creatively address constraints andsimultaneously increase generativeness and robustness.

Therefore, there is a research gap: can we identify those critical features of design processes through which constraints might lead simultaneously to more generativeness and more robustness?

\section{The strong tropism of design tools and hypothesis formulation}

We do not study every feature of the entire NPD process in this study. Instead, we focus on one specific aspect: software used to support NPD and, more precisely, software used at the design gap. This focus helps us formulate more specific research questions and leads us to use a relevant methodology. Let us first briefly review design tools and how they contribute to the generativeness and robustness of the design process in which they are used.

\section{Tools providing high generativeness and poor robustness}

The first category of tools offers a high capacity for conceptual exploration - at little or no cost - but is not integrated into the industrial process, which can result inthe loss of design intentionsduring the production process. These tools are based on sketching and tend to share most of the properties inherent in sketching: they are quick, timely and inexpensive. Sketching itself is obviously one of these tools, but we can also consider clay modeling and patchworking as belonging to this category because they are characterized bycertain properties and uses during the early phases of the design process. These tools aim to provide maximum creativity to their users and are frequently used to present a set of possibilities at the beginning of a design process (Christiansen et al., 2010). These tools are also well suited for introducing rapidly formalized concepts and are considered an explorative method, which makes them consistent with the generativeness of the design process. Sketching makes mental models easy to represent and manipulate, and its simplicity aids designers in reinterpretations and discussions with others(Van Der Lugt, 2002); moreover,sketchingcan be used as a communication tool for conveying an industrial designer's ideas or concepts to other designers, engineers or managers, as noted by(Perks et al., 2005).

In addition to traditional "sketching" tools, digital design interfaces that mimic such traditional tools have appeared (Bae et al., 2008) that have the same advantages as traditional tools, such as good support for creativity (Barone, 2004), but that also share the flaws of traditional tools. These "digital sketching" tools provide poor integration, primarily because 3D models are not compatible with CAD industrial 
environments, and they contribute little to a design's robustness. These toolsare not assessed in this paper because they do not qualify as integrated. Thus, in this category of tools, robustness is traded for maximum generativeness.

Tools providing high robustness and poor generativeness

As numerous industrial tools were progressively introduced into design environments, the first generation of CAD tools gave creative professionals such as industrial designers (IDs) the opportunity to use the same means of operation as engineers. However, although these tools provide good integration, they have the unwanted tendency to sacrifice creativity by restraining the possibilities of conceptual exploration. This category of tools tends to bindcreativity and overliesupon original concepts by integrating them early in the process through technical and even legal constraints.

Theselimitationsresult because these tools were first designed for engineers(Henderson, 1998), and their first intended use was to increase the quality and robustness of designs by limiting the most costly iterations indifferent media (such as blueprints, prototypes and 3D models). Like sketches, the first generation of CAD tools proved to be very good coordination and collaborative tools that had the advantage of providing unambiguous product representations(Thomke, 2003). These tools could generate "boundary-objects" and had good capabilities for transferring, translating and transforming knowledge across syntactic, semantic and pragmatic boundaries (Carlile, 2002, Carlile, 2004)among both IDs and other design participants (e.g., managers, engineers, marketers, etc.). However, along with these qualities, the first-generation CAD tools had several drawbacks that limitedcreativity: limitations on modeling possibilities that led to circumscribed thinking, premature fixation that required detailed modeling too early in the process and bounded ideation (Robertson et al., 2007). IDs also complained about thelack of control and spontaneity with these tools and felt that their intuitive design qualities were transformed into virtual data processing(Wendrich et al., 2009). Computers compartmentalize, break activities into isolated steps and focus on rigid logic and literal meanings(Diffrient, 1994); when using these tools, IDs also tended to focus on geometrical aspects and neglectmeaning creation (Verganti, 2008), one of their essential competencies. Thus, this type of tool trades generativeness for maximum robustness.

The first generation of digital tools thus restricted creative designers' choices: on the one hand, there were creative tools, such as sketching, that were not well-suited to industrial requirements because they traded robustness for generativeness. On the other hand, effective integrative tools for managing collaboration and industrial constraints bounded (or obliterated) ideation and thus effectively traded generativeness for robustness. This literature review of IDs' design tools seems to indicate a strong split related to the tradeoff between robustness and generativeness.

Hypothesis formulation

To confirm the properties of the first generation of CAD tools with respect to design tradeoffs, we formulate our first working hypothesis.

- H1: When designers use first-generation CAD tools, they can improvethe robustness of concepts, but at the expenseof generativeness. 


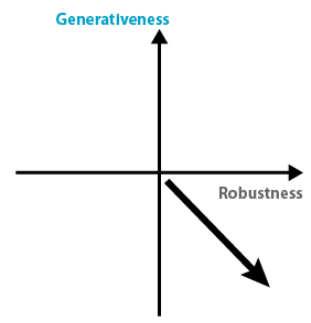

Figure 2. H1 mapped in the robustness/generativeness space

In this context, it was difficult to find tools that can simultaneouslyaddress robustness and generativeness. Today, however, a new generation of digital tools has integrated itself into product design software suites (andare effective regarding robustness, as were the first-generation CAD tools) that claim to maintain designers' abilities to naturally shape objects by following the logic of clay modeling or sketching in a virtual environment. This new generation of tools tends to increase robustness and generativeness, as some of its users claim. To assess the capacity of the new generation of CAD tools to simultaneously increase robustness and generativeness, we formulate our next working hypothesis.

- H2: When designers use newer generations of CAD tools, they are able to simultaneouslyimprove the robustness and generativeness of concepts.

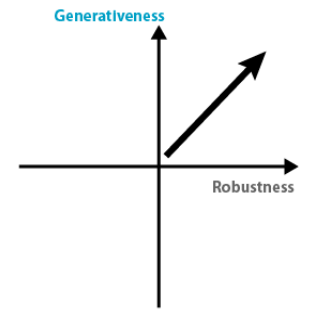

Figure 3. H2 mapped in the robustness/generativeness space

This result is the first step in showing that there is not necessarily a conflict between constraints (represented by an increase in robustness) and creativity (represented by an increase in generativeness). If $\mathrm{H} 2$ is confirmed, we study the new-generation CAD tools to understand how software assists in creatively addressing constraints. In particular,we aim to discover whether there is a detailed causal relationship between an increase in constraints (i.e., an increase in robustness) and an increase in generativeness.In addition, we also attempt to discover the phenomenon that helpsus use constraints in a generative way.Thus, our third hypothesis is as follows:

- H3: The new generation of CAD tools accounts for more constraints and helps designers to creatively address those constraints.

\section{Experimental method}

To test our hypotheses, we rely on an experimental method. Below, we present the experimental protocol (task, sample, observations, etc.). 


\section{Choice of design step and tools}

The basic idea of the experiment consists of measuring how two different CAD tools lead to two different types of performance in terms of generativeness and robustness.

The design task. We focus on one specific task in the entire development process: the so-called ideation gap (or design gap). This phase is one of the most emblematic in the design process, namely, the transition between "traditional" and "numerical" media (Wendrich et al., 2009). At this brutal and stringent transition point, the task consists of taking concepts represented using traditional means (sketches, mock-ups, prototypes) and modeling (digitizing) them on computers. This step is stressful for the software and exposes bothits limitations and capabilities. This transition is critical because there is a risk of losingthe initial design intentions during the switch from traditional to digital media. The integration of concepts into the software design suite is intended to support the subsequent development of the design into the final product. Thus, after the design gap, robustness should be increased and industrial constraints should be addressed, but the greatest threat is that these objectives will be achieved at the expenseof a loss in generativeness.

We compare two software packages used by designers on a daily basis to bridge the design gap. Both packages integrate handmade sketches into the same software design suite and seek to satisfy certain modeling constraints. Each design tool is a so-called "workshop" that helps the designer transform a handmade sketch into a digital shape, as illustrated in Figures 5 and 6.

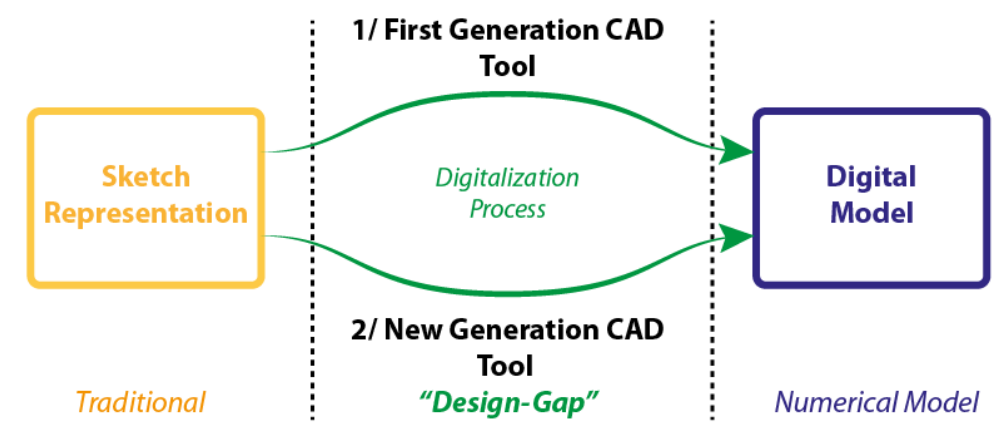

Figure 4. Managing the design gap with two different digital tools

The first workshop that we tested is an archetypal first-generation CAD tool. This tool uses procedural commands, and modeling appears as a succession of steps in which blueprint construction (called twodimensional (2D) sketches in the software) is followed by employing parameterizable functions (such as extrusions, revolutions or sweeps) in iterations. The tool is capable of producing very high quality surfaces (up to a Class A standard, which is the highest in the industry).

The new-generation CAD toolsprovide integration within the same design suite. The global design suite is suitable for any type of industrial design and is composed of specialized workshops (i.e., other design tools for specific tasks). The models generated within the suite are fully compatible with various workshops. Any creative design performed with either tool can then be transmitted to other designers and has the capacity to integrate industrial constraints.

\section{Design briefs used in the experiment}




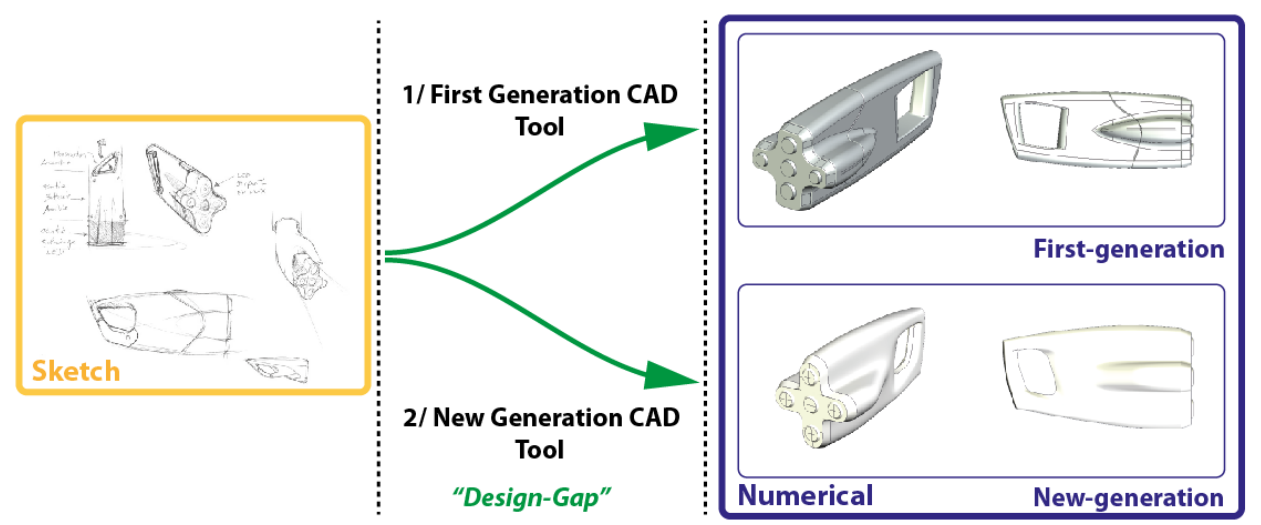

Figure 5. Materials collected before and after the design gap

IDs in most companies typically cross the design gap with first-generation CAD tools because they serve as direct entry points into the industrial world and its CAD codifications and specifications, which are required for subsequent manufacturing processes. Our goal is to assess the capabilities of recently introduced new-generation tools and to compare them with their predecessors to determine whether these new tools can verify $\mathrm{H} 2$.

Sample: For the experiment, we worked with six IDs from a well-known CAD company. All the participants had ID educations and had worked as designers for three to 20 years using these two types of tools. These users had experimented with the tools. In this way, we avoid a bias linked to experience with the tools and in-experiment learning processes.

The IDs were given design briefs thatdescribed precisely what was expected of them.

- In the first step, the IDs were asked to produce two different concepts of an "autonomous portable lamp" and to represent them with sketches. Each of the IDs was given full access to ideation materials: pen, paper, pencils, erasers and a computer with graphing software. They had one hour to complete their designs and were free to ask questions. When they were finished, their sketches were collected and scanned.

- Next, the IDs were asked to make 3D models of their sketched concepts with both the first- and newgeneration CAD modeling tools. They had one hour for each concept model, i.e., a total of two hours. The IDsactions were recorded throughout the experiment. To avoid any learning effects, we randomized the modeling: half of the IDs began with the first-generation software, and the other half beganwith the newgeneration software.

\section{Data and evaluation protocol}



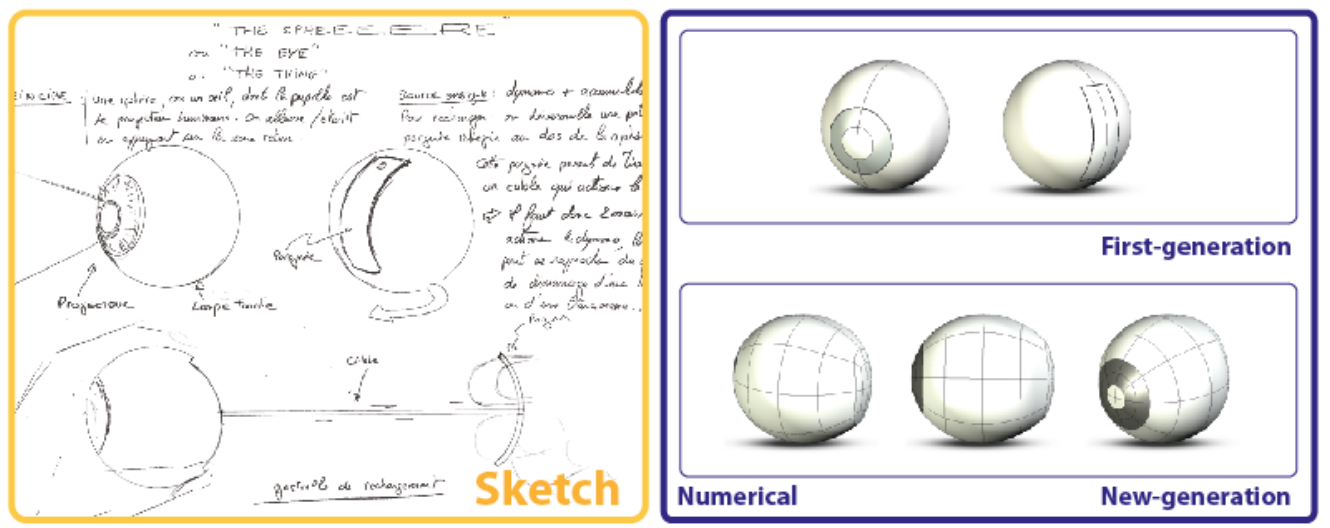

Figure 6. Example of a sketch (before the design gap) and CAD representations (after the design gap; with first-generation CAD tool (first line) and second-generation CAD tool (second line) (representations collected for Concept 4)

For each sketched concept, we obtained two sampled numerical representations, which resulted in 24 representations. Figure 7 shows a collection of sketches and 3D models for one concept.

For an assessment of their respective contributions to design robustness and generativeness in the design gap, originality and feasibility items were scored according to the literature (Runco and Charles, 1993, Magnusson, 2003). The formal originality of a concept is consistent with the generativeness of a design. A highly original shape is a guarantee of high creative potential, which is available for exploration and innovation throughout the design process. This originality of design is uncommon and surprising and can reveal new meanings. The formal feasibility of a concept is similar to the robustness of a design, i.e., its acceptability during the remainder of the design process. A feasible shape enablesa simplified design with fewer unknowns and difficulties; in particular,it has a given quality of surface, which can be rated and evaluated using mathematical and optical criteria. When interviewing evaluation experts after the experiment, they confirmed that they considered a design to be robust when it met the constraints listed above (a digital shape that can be handled by CAD software suites - throughout the remainder of the development process - and that meets "quality" criteria, i.e., it is (likely) to be feasible and simple, and more precisely, to avoid any optical or other types of defects). The evaluation experts also confirmed that they considered a shape to be "original" when it was different from what they expected but that also seemed to be iconic, exact and simple.

We provide a graphical example of what is called surface quality. Along with visual criteria, it is also possible to describe the quality of a shape in mathematical terms (see Figure 8). 
Surface of good quality
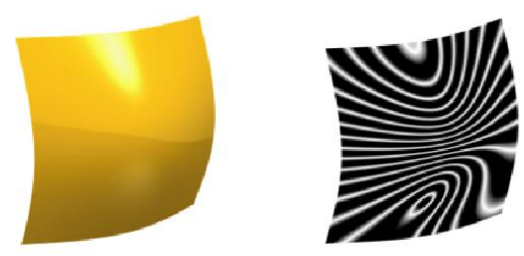

Surface of poor quality

Tangency discontinuity

Curvature discontinuity
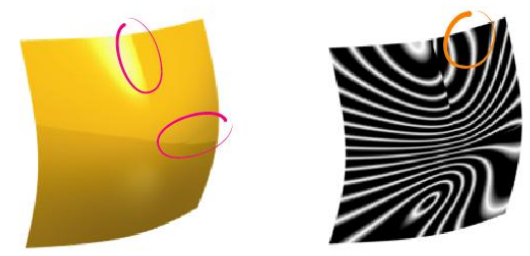

Figure 7. Evaluation of surface quality

To evaluate the evolution of feasibility and originality during the design gap, we used an expert evaluation, the "Consensual Assessment Technique" (CAT), developed by Amabile et al. (Amabile, 1996). We selected five different IDs who were experienced in assessing design concepts and worked as managers. These IDs were used to rapidly evaluate projects under development; in the remainder of this article, these IDs are referred to as "experts". To enablethese experts to evaluate the evolution of feasibility and originality, we provided them with the reference sketch and the pair of digital 3D models for each concept. The experts rated the progression of feasibility and originality of the concept's shape from the sketch to the digital model using a Likert scale (Likert, 1932) of five items: strong decrease (-2), decrease (-1), neutral (0), increase (+1) and strong increase (+2). The experts conducted this process twice, once for the first-generation CAD tool model and again for the new-generation CAD tool model.

Ultimately, we obtained two evolution grades for each concept and for each type of modeling tool, i.e., one for feasibilityevolution and the other for originality evolution. With 12 concepts and 24 numerical models, the experiment yielded 48 evolution grades.

To assess the respective impact of each CAD tool on concept feasibility and originality, we calculated the progression for each modeled concept property as the mean of all five experts' scores. The result is an aggregated $\Delta$ Originality $(\Delta \mathrm{O})$ and a $\Delta$ Feasibility $(\Delta \mathrm{F})$ for each numerical concept that is matched with the tool used. This result can be mapped on the $\Delta \mathrm{F}-\Delta \mathrm{O}$ space and provides a designgap performance measure for a single concept, depending on the tool used.

\section{Results}

\section{Quantitative results (H1 and H2)}

As described above, we characterized the design gap performance of the tools and mapped it in the $\Delta \mathrm{O}-$ $\Delta \mathrm{F}$ space. When the experts rated a strict progression of originality and feasibility $(\Delta O<0$ and $\Delta F>0)$, the concept had become simultaneously more original and feasible after the design gap. We call this result a simultaneous solution. Withreduced originality and increased feasibility ( $\Delta O \leq 0$ and $\Delta F \geq 0$ ), the concept gains feasibility, but at the expense of its initial originality. We call this result a trade-off. In other configurations, there is a strict decrease in feasibility $(\Delta F<0)$, which might indicate a gain ("Other1") or a 
loss ("Other2") in originality. The results of the evaluations of the progressions are illustrated in Figure 10 for each tool.

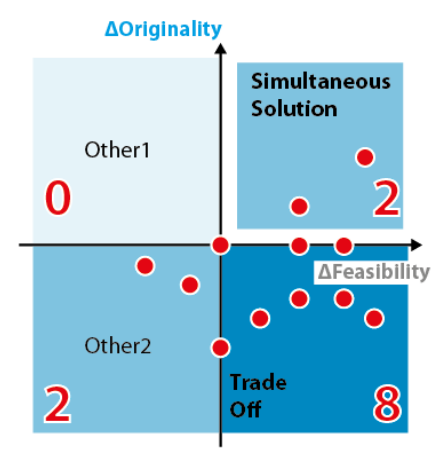

First-generation CAD Tool

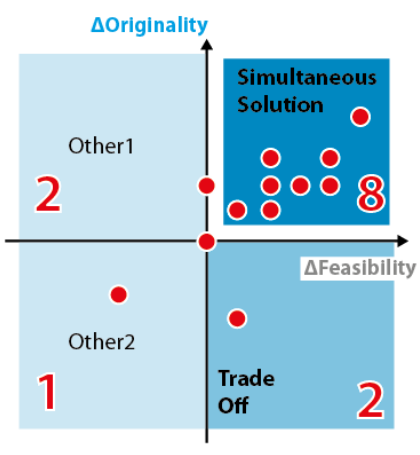

New-generation CAD Tool

- = Design-Gap performance for one modeled concept

Figure 8. Map of the design gap performanceof first-and new-generation CAD tools

The results are visually eloquent, but we now test our hypotheses statistically.

H1-0 hypothesizes that the design gap performance of the first-generation CAD tool leads to a uniform distribution among the four different sectors:trade-off, simultaneous solution, "Other1" and Other2." We test H1-0 with a $\chi^{2}$ test for a two-sided $2.5 \%$ trust interval. We obtain a total distance of 12 for the $\chi^{2}$, which is superior to the table distance of 9.3. Thus, we reject H1-0 and formulate a non-uniform distribution of design gap performance for first-generation CAD tools. We next test whether there is a significant difference among the proportions of tradeoff, simultaneous solution, "Other1" and Other2." A difference is evident in tradeoff occurrences, ascan be observed by examining the two-sided 5\% confidenceinterval: with 8 of the 12 concepts, the frequency of tradeoffis $66.7 \% \pm 22.3 \%$, whereas for simultaneous solution, the frequency is $13.7 \% \pm 17.6 \%$. Thus, tradeoff frequency is significantly superior to simultaneous solution frequency (and "Other1" and "Other2"). Therefore, we conclude the following for H1: when IDs use first-generation CAD tools, they improve the formal feasibility of concepts at the expense of formal originality.

H2-0 hypothesizesthat the design-gap performance of the new-generation CAD tool leads to a uniform distribution among the four different sectors:trade-off, simultaneous solution, "Other1" and Other2." We test H2-0 with a $\chi^{2}$ test for a two-sided $2.5 \%$ confidenceinterval. We obtain a total distance of 11.3 for the $\chi^{2}$, which is superior to the table distance of 9.3. Thus, we reject $\mathrm{H} 2-0$ and formulate a non-uniform distribution of design gap performance for the new-generation CAD tool. We next test whether there is a significant difference among the proportions of tradeoff, simultaneous solution, "Other1" and Other2." A difference is evident in simultaneous solution occurrences, as is shownin the two-sided 5\% confidence interval: with 8 of the 12 concepts, the frequency of simultaneous solution is $66.7 \% \pm 22.3 \%$. For tradeoff, the frequency is $13.7 \% \pm 17.6 \%$. Thus, the simultaneous solution frequency is significantly superior to the tradeoff frequency (and "Other1" and "Other2"). Therefore, with respect toH2, we conclude as follows:when IDs use the new-generation CAD tool, they can simultaneously improve concepts' formal feasibility and originality.

\section{Qualitative results (H3)}


Now that we have shown an (unexpected) relationship between robustness (constraints) and generativeness (creativity), we analyze these phenomena in more depth. In particular, we underline, in the theoretical framework, that a design theory perspective reveals the existence of two competing processes when a designer addresses constraints: on the one hand, constraintslower degrees of freedom, tending to decrease the capacity for originalityand generativeness; on the other hand, constraints can push a designer to design an unexpected outcome, which could indicate that a constraint might increase generativeness.

In our experimental study, how do these two processes unfold? We clarified this unfolding by means of an in-depth study of the use of CAD tools by designers and complementary interviews with users.

1- Studying the software in more detail, it appears that both software suites help meet the CADcompatibility constraint: any shape coded with either software suite can be coded in CAD to be used in the rest of the CAD suite. The second generation integratesan additional constraint: the software permits any shape created by the user to meet the optical quality criteria. This result derives from the software principle: in the second-generation software, an initial simple rough shape is provided, and the user deforms it step by step into a new shape.The initial shape meets the relevant optical quality, and every transformation is coded such that the resulting shape also follows the optical quality criteria (this resultsbecause the surface always follows a certain curvature continuity). Thus, the first-generation software tends to embody fewer constraintsthan the second-generation software.

2- This property of the second-generation software has an immediate consequence: with the secondgeneration software, is it impossible to design a shape that would not follow the optical criteria. Thus, there is a restriction in the degree of freedom left to the users. All the users and software designers confirmed this property: every shape that can be created with the second-generation software can be made with the first-generation software, whereas some shapes that can be made with the first-generation software cannot be made with the second-generation software. There are clearly fewer degrees of freedom in the second-generation software. Thus, the experiment follows the first process: more constraints lead to fewer degrees of freedom.

3- Conversely, the users also explain that manipulation of the modeled objects in the secondgeneration software is direct and provides instant feedback via a "manipulation box." This process allows for a high degree of precision in creating and modifying shapes. Software users can work on shapes with substantial control and speed without invoking commands, functions or parameters. Notably, users sometimes refer to this tool as a type of clay modeler.Thus, they are able to visualize many shapes that follow the constraints. Furthermore, in this process, they are able to consider the "usual" shapes that could be obtained following the constraints andto explore whether there were "unusual" shapes that follow the constraints but diverge from expected shapes. Here, we understand how the second (competing) process occurs: by enabling users to play with constraints, the software helps visualize "expected" shapes andhelps the user to design an unexpected shape to meet the criteria. Embedding the constraints diminishes the degree of freedom but can help users to depart from the "expected" shapes.

Thus, this study confirms hypothesis H3: The new generation of CAD tools takes more constraints into account and help designers creatively address those constraints.

With the second-generation software, quality constraintstrigger creativity. This finding may appear surprising at first, but the literature on multiple domains is characterized bymany examples of creativity increased by constraints, as brilliantly illustrated by G. K. Chesterton: "Art consists of limitation. The 
most beautiful part of every picture is the frame." What we add to this illustration is that the tool itself (not only the designer) embodies the constraint. The software tools that help the designer to simultaneously increase robustness and originality are actually engines that help the designer play with constraints in a generative way - these enginesenable the designer to creatively address constraints.

This result has an interesting consequence: the originality of the final shape is deeply linked to thevalidated optical criteria. Thus, one can consider that the originality is validated as well. The two dimensions, feasibility and originality, are now coupled together in a positive manner: if maintaining the optical quality is an objective, then the shape and the originality associated with it must be preserved. Conversely, reducing shape originality does not increase robustness but instead decreases the optical quality anddecreases robustness. Freezing the degree of freedom of a shape increases robustness and increases originality. Thus, one can speak of "robust originality" or "acquired originality."
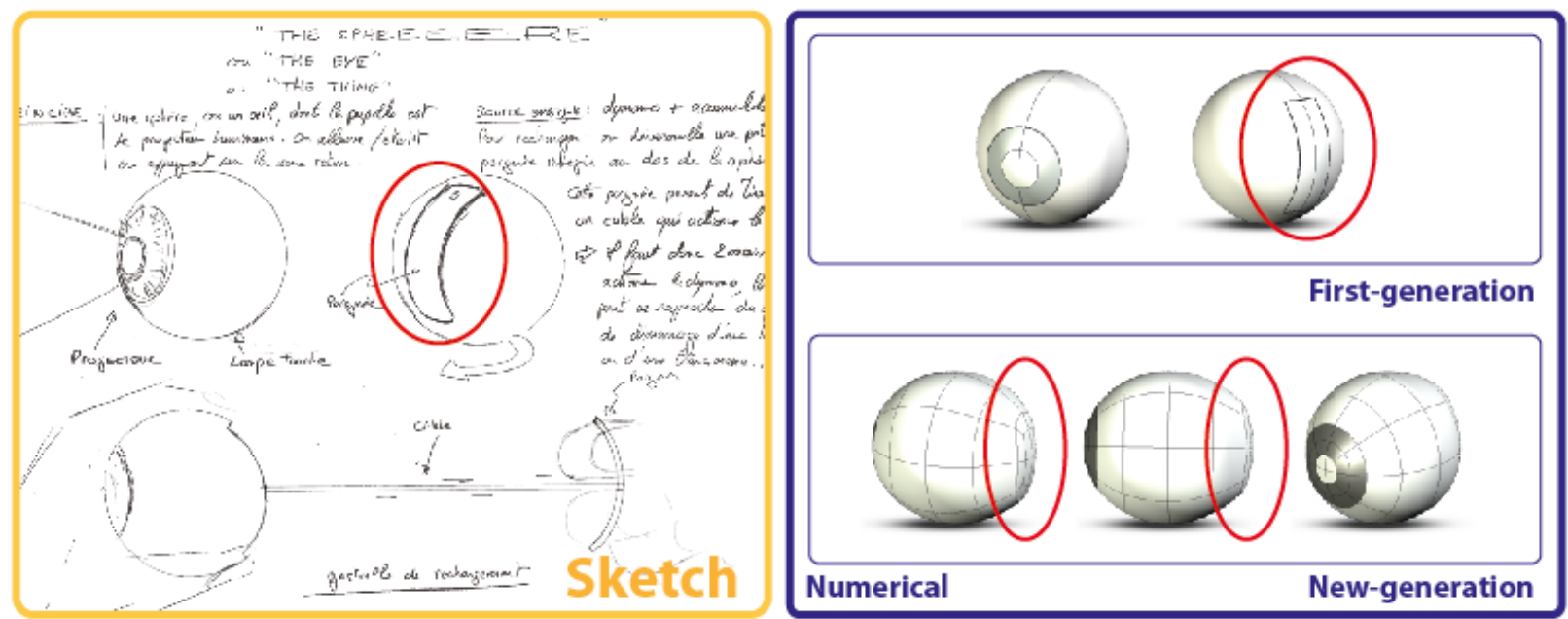

Figure 9. Appearance of a new shape attribute using the new-generation CAD tool

This conceptis illustrated above (see Figure 10): the first generation software transforms the initial sketch into an ovoid shape. The ovoid surface actually corresponds to what is typically expected to follow the optical quality criteria (the evaluation is as follows: "less originality, more robustness"). With the secondgeneration software, the final shape is evaluated as having "more originality and more robustness". The experts justifytheir evaluations by explaining that a facet appears on the ovoid shape. Because that shape is coded by the second-generation software, the user is sure that it will meet the optical quality criteria. However, the shape is more surprising and original than the almostperfectly spherical model. The designer can alter the spherical design while maintaining optical quality, addinga facet that was barely present in the original sketch and that is not typically associated with A-level optical quality (A-level optical quality favors strong surface continuities, whereas facets tend to introduce discontinuities). The constraint led to originality, and the originality was acquired jointly with robustness.

\section{Discussion and Further Research}

\section{Limitations}


Due to the exploratory nature of this experiment, our findings have certain limitations. The first restriction concerns the limited number of representations, concepts and IDs used in the experiment. This limitationmay have seriously misleading effects, although our results seem to indicate a global trend that supports our findings. The design of the experiment (a comparative empirical study that resulted in paired samples) helped achieve a high confidence level despite its small sample size. The shape originality and feasibility offered by the modeling tools might be correlated with the type of concept, which in our case was an "autonomous portable lamp." It would be useful to conduct our experiment with several concepts depicting various products from different industries.

\section{Side Findings and Further Developments}

Sketching is not the only representation that IDs use for concept exploration. They also frequently manipulate clay models, 3D digital models made with "3D artist"-type tools, prototypes and even photomontages. It would be useful to measure the progression of shape originality and feasibilitywhen transitioning from those types of media to 3D industrial models.

In future work, we propose varyingexperts' evaluation methods by providing them with efficient 3D viewers prototyped to enable a different perception of numerical models. We also note that the time taken by the designers to model the different representations of their concept was correlated strongly with the tools that they used. First-generation CAD tool modeling took approximately $40 \%$ longer than modelingwith the new-generation $\mathrm{CAD}$ tool. In addition to these tools' respective contributions to product design, it would beinteresting to assess their productivity. We also plan to obtain a deeper understanding ofacquired originality and to attempt to model it, along with how it might be obtained in different contexts.

\section{Conclusions and Managerial Implications}

\section{Conclusions}

The results of this experiment include several new findings and confirm certain results in the literature. The dominant industrial tools, i.e., software similar to the first-generation CAD tool used in this experiment, have a powerful capacity to dramatically improve the robustness of a design, but at a the expense of its generativeness. By contrast, the new-generationCAD tool providesthemeans to increase robustness andgenerativeness. Moreover, we have shown that this property arisesbecause the tool is an engine that can be used to explore a variety of ways to address constraints and to depart from the "usual" shapes, which thus increases originalitywhile meeting constraints.

\section{Managerial Implications}

With procedures such as those in the new-generation CAD tools and their capacity to simultaneously enhance generativeness and robustness during design processes, designers may be able to manage the generativeness and robustness of their designs to best fit the needs of their companies at any given moment. With such capabilities, the design process could be revised, and its versatility and robustness could be improved markedly, as illustrated in Figure 11. 


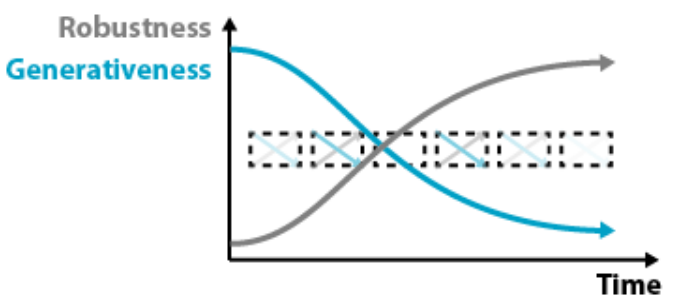

"Standard" Design process

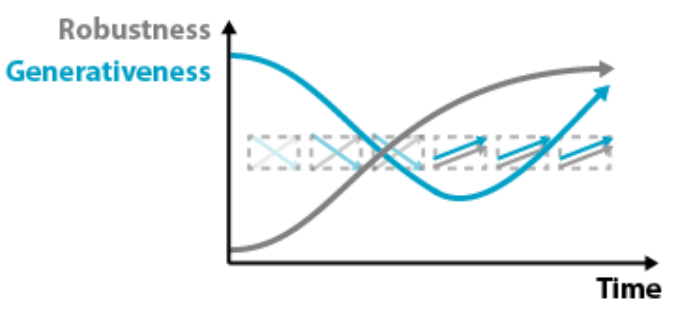

"New" Design process

Figure 10. "Standard" and "New" design process profiles

With such a tool, it is not necessary to begin with a high originality level. It is possible to increase generativeness even when robustness must be increased. This experiment also suggests that because tools are now able to simultaneously enhance a concept's robustness and generativeness, a new design process can be imagined and applied in industry. This process would have properties other than tradeoffs and offer designers the capacity to add robustness and/or generativeness as required.Thus, the new design tools' ability to creatively address constraints paves the way for new innovation and creativity management processes.

\section{Acknowledgments}

Allthe participating industrial designers and experts took time from their work to participate in this experiment. We thank them for their time, consideration and effort; we also thank the CAD company that hosted us when we performed the experiments.

We thank the reviewers and the editor of the special issue for their fruitful comments and suggestions.

\section{Bibliography}

AMABILE, T. M. 1998. How to kill creativity. Harvard Business Review, september-October 1998, 77-87.

BAE, S.-H., BALAKRISHNAN, R. \& SINGH, K. ILoveSketch: As-natural-as-possible sketching system for creating 3D curve models. ACM Symposium on User Interface Software and Technology, October 19-22, 20082008 Monterey, CA.

BARONE, M. The Process Improvement Impact on the Styling Workflow of an Industrial Design Company. In: HUGHES, J. F. \& JORGE, J. A., eds. Eurographics Workshop on Sketch-Based Interfaces and Modeling, 2004. 19-25.

BODEN, M. A. 1990. The creative mind. Myths and Mechanisms, George Weidenfeld and Nicolson Ltd.

BUIJS, J. 2003. Modelling Product Innovation Processes, from Linear Logic to Circular Chaos. Creativity and Innovation Management, 12, 76-93.

BURKHARDT, J.-M. \& LUBART, T. 2010. Creativity in the Age of Emerging Technology: Some Issues and Perspectives in 2010. Creativity and Innovation Management, 19, 160-166.

CARLILE, P. R. 2002. A Pragmatic View of Knowledge and Boundaries: Boundary Objects in New Product Development. Organization Science, 13, 442-455.

CARLILE, P. R. 2004. Transferring, Translating, and Transforming: An Integrative Framework for Managing Knowledge Across Boundaries. Organization Science, 15, 555-568.

CHRISTIANSEN, J. K., VARNES, C. J., GASPARIN, M., STORM-NIELSEN, D. \& VINTHER, E. J. 2010. Living Twice: How a Product Goes through Multiple Life Cycles*. Journal of Product Innovation Management, 27, 797-827. 
COUGER, J. D. 1990. Ensuring creative approaches in information system design. Managerial and Decision Economics, 11, 281-295.

DIFFRIENT, N. 1994. Confessions of a RELUCTANT TECHNOLOGIST. Design Management Journal (Former Series), 5, 83-85.

DORST, K. \& CROSS, N. 2001. Creativity in the design process: co-evolution of problem,Äìsolution. Design Studies, 22, 425-437.

FUJIMOTO, T. 1997. Shortening Lead Time through Early Problem Solving, a new Round of Capability Building Competition in the Auto Industry. In: JÜRGENS, U. (ed.) New Product Development and Production Networks, Global Industrial Experience. Berlin: Springer.

GOLDENBERG, J. \& MAZURSKY, D. 2000. First We Throw Dust in the Air, then We Claim We Can't See: Navigating in the Creativity Storm. Creativity and Innovation Management, 9, 131-143.

HATCHUEL, A. 2002. Towards Design Theory and expandable rationality: the unfinished program of Herbert Simon. Journal of Management and Governance, 5, 260-273.

HATCHUEL, A., LE MASSON, P., REICH, Y. \& WEIL, B. A systematic approach of design theories using generativeness and robustness. International Conference on Engineering Design, ICED'11, 2011 Copenhagen, Technical University of Denmark. 12.

HATCHUEL, A. \& WEIL, B. 2009. C-K design theory: an advanced formulation. Research in Engineering Design, 19, 181-192.

HENDERSON, K. 1998. On Line and On Paper, Visual Representations, Visual Culture, and Computer Graphics in Design Engineering, MIT Press.

KARNIEL, A. \& REICH, Y. 2011. Managing the dynamics of Nex Product Development Processes - A new Product Lifecycle Management Paradigm, London, Springer.

KHURANA, A. \& ROSENTHAL, S. R. 1998. Towards Holistic "Front Ends" In New Product Development. Journal of Product Innovation Management, 15, pp. 57-74.

LE MASSON, P., HATCHUEL, A. \& WEIL, B. 2011. The Interplay Between Creativity issues and Design Theories: a new perspective for Design Management Studies? Creativity and Innovation Management, 20, 217-237.

MAGNUSSON, P. 2003. Managing User Involvement for Service Innovation, findings from end-user telecom services innovation. Journal of Service Research, 6, 111-124.

MOEHRLE, M. G. 2010. MorphoTRIZ - Solving Technical Problems with a Demand for Multi-Smart Solutions. Creativity and Innovation Management, 19, 373-384.

OLSON, E. M., COOPER, R. \& SLATER, S. F. 1998. Design strategy and competitive advantage. Business Horizons, 41, 55-61.

PERKS, H., COOPER, R. \& JONES, C. 2005. Characterizing the Role of Design in New Product Development: An Empirically Derived Taxonomy*. Journal of Product Innovation Management, 22, 111-127.

REID, S. E. \& DE BRENTANI, U. 2004. The Fuzzy Front End of New Product Development for Discontinuous Innovations: A Theoretical Model. Journal of product innovation management, 21, 170-184.

RITTEL, H. W. J. 1972. On the Planning Crisis: Systems Analysis of the 'First and Second Generations'. Bedriftsokonomen, 8, 390-396.

ROBERTSON, B. F., WALTHER, J. \& RADCLIFFE, D. F. 2007. Creativity and the Use of CAD Tools: Lessons for Engineering Design Education From Industry. Journal of Mechanical Design, 129, 753-760.

RUNCO, M. A. \& CHARLES, R. 1993. Judgments of originality and appropriateness as predictors of creativity. Personality and Individual Differences, 15, 537-546.

SCHÖN, D. S. 1983. The Reflective Practitioner, How Professionals Think in Action, Basic Books Inc., USA.

SCHÖN, D. S. 1990. The Design Process. In: HOWARD, V. A. (ed.) Varieties of Thinking. Essays from Harvard's Philosophy of Education Research Center. New York, NY: Routledge. 
SEIDEL, V. P. 2007. Concept Shifting and the Radical Product Development Process. Journal of product innovation management, 24, 522-533.

STERNBERG, R. J. \& LUBART, T. I. 1999. The Concept of Creativity: Prospects and Paradigms. In: STERNBERG, R. J. (ed.) Handbook of Creativity. Cambridge: Cambridge University Press.

SUH, N. P. 1990. Principles of Design, New York, Oxford University Press.

THOMKE, S. H. 2003. Experimentation Matters. Unlocking the Potential of New Technologies for Innovation, Boston, Harvard Business School Press.

THOMKE, S. H. \& FUJIMOTO, T. 2000. The Effect of "Front Loading" Problem-Solving on Product Development Performance. Journal of Product Innovation Management, 17, pp. 128-142.

ULLMAN, D. G. 1997. The Mechanical Design Process, Boston, MA, Mc Graw Hill.

VAN DE VEN, A., POLLEY, D. E., GARUD, R. \& VENKATARAMAN, S. 1999. The Innovation Journey, NewYork, Oxford, Oxford University Press.

VAN DER LUGT, R. 2002. Brainsketching and How it Differs from Brainstorming. Creativity and Innovation Management, 11, 43-54.

VERGANTI, R. 2008. Design, Meanings, and Radical Innovation: A Metamodel and a Research Agenda. Journal of product innovation management, 25, 436-456.

WENDRICH, R. E., TRAGTER, H., KOKKELER, F. G. M. \& VAN HOUTEN, F. J. A. M. 2009. Bridging the Design Gap: Towards an Intuitive Design Tool. 26th ICSID World Design Congress and Education Congress.

YOSHIKAWA, H. 1981. General Design Theory and a CAD System. In: SATA, T. \& WARMAN, E. (eds.) ManMachine Communication in CAD/CAM, proceedings of the IFIP WG5.2-5.3 Working Conference 1980 (Tokyo). Amsterdam, North-Holland. 